\title{
MicroRNA knocks some sense into senseless
}

\begin{abstract}
MicroRNA-9a (miR-9a) is involved in the rumble to regulate neurogenesis, a recent paper in Genes \& Development shows. In Drosophila melanogaster, sensory organ precursors (SOPs) - which go on to generate sensory organs and
\end{abstract}

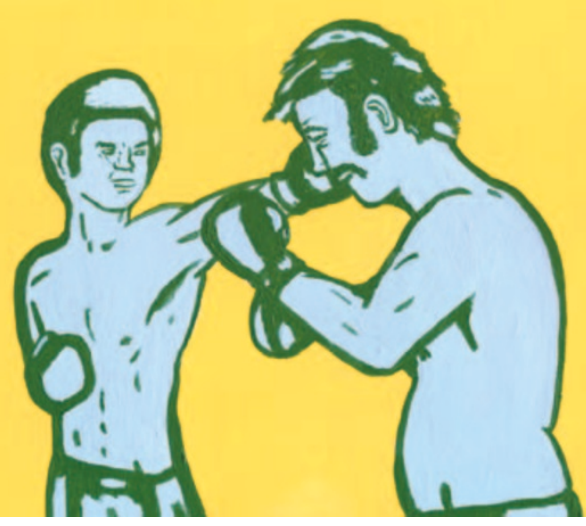

neurons - develop from cells with the highest proneural gene expression. Until recently, it was thought that positive feedback generated by senseless (sens) coupled with lateral inhibition mediated by Notch and Delta was enough to generate differential proneural gene expression and SOP development. But the Gao group now shows that $m i R-9 a$ offers a new layer of regulation $-m i R-9 a$ regulates sens expression through its $3^{\prime}$ untranslated region ( $3^{\prime}$ UTR) and ensures the precise specification of SOPs.

Gao and colleagues first generated miR-9a-deficient $D$. melanogaster strains. These strains were viable and fertile, but larvae and adults had an abnormal morphology. Roughly $40 \%$ of mutant larvae had additional sensory neurons that were derived from SOPs. Similarly, 40\% of mutant adults had additional sensory bristles on their anterior wing margins, $\sim 15 \%$ had additional sensory organs on their notums, and $100 \%$ had posterior wing margin defects. By contrast, flies that overexpressed $m i R-9 a$ had markedly reduced numbers of sensory organs on both their wings and their notums.

Interestingly, the wing margin phenotype of flies that were deficient in $m i R-9 a$ was similar to that of flies with a gain-of-function mutant of sens and that of flies in which sens was overexpressed. High-resolution in situ hybridization in the wings of control flies showed that miR-9a is not expressed in SOPs, and in vivo immunostaining of the dorsal region of the wing and the notum showed that overexpression of $m i R-9 a$ corresponded with suppressed expression of sens.

To further investigate the relationship between sens and miR-9a, the 3' UTR of sens was placed downstream of a reporter gene. Strikingly, co-expression of this construct with

\section{Independent partners}

Planar cell polarity (PCP) controls the orientation of epithelial cells in animals and plants. Reporting in Development, Casal, Lawrence and Struhl now challenge the prevailing model for PCP in Drosophila melanogaster.

The existing model for PCP establishment in D. melanogaster proposes that a morphogen gradient organizes the expression of two atypical cadherins, Dachsous (Ds) and Fat (Ft), and of the Golgi protein Four-jointed, to set up Ds-system gradients. These then function through the Stan system, which includes the cadherin receptorlike molecule Starry Night (Stan) and the Wnt receptor Frizzled (Fz), to orientate hairs and ommatidia.

The authors used the abdomen as an experimental model, in which - in contrast to the eye - the polarity of each cell is shown directly by the orientation of hairs that are produced only by this cell. The authors used genetic mosaics to show that the Ds system can initiate and propagate PCP, even when the Stan system is not functional. For example, they showed that ectopic expression of Ds repolarizes surrounding cells, even in flies that lack both Stan and Fz.

If these two systems are independent, then the loss of both systems should cause more damage than the loss of either system. Indeed, the hair polarity of $d s^{-} \operatorname{stan}^{-}$flies is randomized whereas polarity is much more normal in either $\mathrm{ds}^{-}$or stan- flies.

The authors then showed that 'sending' cells can change the polarity of 'receiving' cells by presenting either Ds or Ft to neighbouring cells.
Both Ds and Ft seem to have ligand-like signalling activities that can repolarize surrounding cells. This signal depends on the ratio of Ds to $\mathrm{Ft}$ in the sending cell. The sending cell does not need both $\mathrm{Ds}$ and $\mathrm{Ft}$ to repolarize nearby cells; however, receiving cells need both proteins to receive and respond. The authors then analysed which domains of Ft and Ds influence the sign of the signal. Analysis of chimeric molecules showed that the nature of the signal of both $\mathrm{Ds}$ and $\mathrm{Ft}$ is governed by their ectodomains.

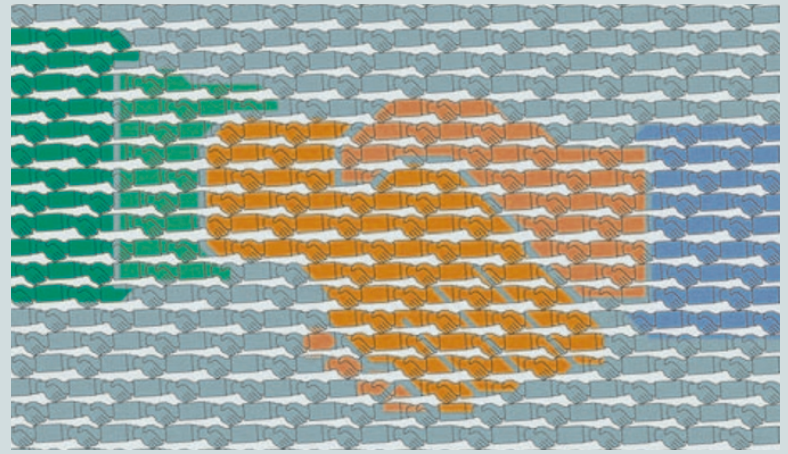


mi9- $\mathrm{Ra}$ reduced the expression of the reporter gene by $70 \%$. So, in cells that are not destined to become SOPs, $m i R-9 a$ inhibits residual sens expression by binding its $3^{\prime}$ UTR, thereby preventing proneural gene expression and SOP development.

But, it seems unlikely that $m i R-9 a$ functions as an absolute switch; only a proportion of $m i R-9 a$-deficient flies developed additional sensory organs. The authors propose that $m i R-9 a$ functions to fine-tune sens expression and the proneural gene feedback loop, thereby ensuring the precision of the production of SOPs. Intriguingly, $m i R-9 a$ is $100 \%$ conserved from flies to humans, and $m i R-9 a$ is highly expressed in neurogenic regions in the developing and adult mammalian brain. Perhaps, the authors note, $m i R-9 a$ is also involved in mammalian neurogenesis.

Asher Mullard

ORIGINAL RESEARCH PAPER Li, Y. et al . MicroRNA-9a ensures the precise specification of sensory organ precursors in Drosophila. Genes Dev. 20, 2793-2805 (2006)

FURTHER READING Cohen, M. S. et al.

Denoising feedback loops by thresholding a new role for microRNA. Genes Dev. 20, 2769-2772 (2006)

According to the linear model of PCP, morphogen gradients, such as Hedgehog $(\mathrm{Hh})$ in the abdomen or Wingless $(\mathrm{Wg})$ in the eye, control polarity by establishing gradients of the Ds system, which then functions through the Stan system. But, if these two systems are independent, how does Hh signalling function? Constitutive activation of the $\mathrm{Hh}$ pathway in clones reverses the polarity of nearby hairs in both $\mathrm{ds}^{-}$and stan ${ }^{-}$flies, but not in $d s^{-} \operatorname{stan}^{-}$flies, which indicates that Hh polarizes cells through both the Ds system and the Stan system.

From these findings, the researchers propose a radical new model that the Ds system and the Stan system each uses an independent mechanism to polarize cells and to propagate polarity from cell to cell.

Ekat Kritikou

ORIGINAL RESEARCH PAPER Casal, J. et al. Two separate molecular systems, Dachsous/Fat and Starry night/Frizzled, act independently to confer planar cell polarity. Development 133 , 4561-4572 (2006)

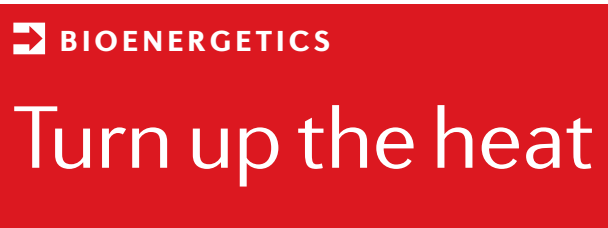

PGC1 transcriptional co-activators are important regulators of mitochondrial biogenesis and respiration. Two new studies from the Spiegelman laboratory now shed light on the role of PGC1 in several disease aetiologies. PGC $1 \alpha$ and PGC1 $\beta$ are implicated in oxidative-damage-related neurodegeneration by suppressing the generation of reactive oxygen species (ROS). Moreover, PGC1 $\alpha$ is associated with defective energy homeostasis associated with Leigh syndrome French Canadian variant (LSFC), a genetic disorder that is characterized by neurodegeneration and severe liver defects.

Given that the mitochondrial electrontransport chain is the main producer of ROS, the authors examined the role of PGC $1 \alpha$ and PGC $1 \beta$ in ROS metabolism. Following exposure to oxidative stress, the expression levels of $\operatorname{Pgc} 1 \alpha$, and to a lesser extent $\operatorname{Pgc} 1 \beta$, were increased, as were those of several genes encoding components of the ROS defence system including several ROS-detoxifying enzymes. RNA-interference-mediated knockdown of either one or both co-activators reduced the basal gene-expression levels of components of the ROS defence system, and the induction of gene expression following an oxidative challenge was practically abolished. Cells from Pgc1 $\alpha$-null mice showed reduced gene expression of components of the ROS defence system and increased sensitivity to oxidative stress. Spiegelman and co-workers therefore concluded that PGC $1 \alpha$ and PGC $1 \beta$ regulate the basal and induced gene-expression levels of the ROS defence system.

By challenging the brain of Pgc1 $\alpha$-knockout mice with oxidative stress, Spiegelman and colleagues observed increased neuronal damage, which occurred with excessive oxidative stress. By contrast, overexpression of PGC1 $\alpha$ in neuronal cells that were subsequently exposed to oxidative stress resulted in increased survival. This protective effect was associated with increased gene expression of ROS-detoxifying enzymes.

These findings imply that PGC1 $\alpha$ "may serve as an adaptive set-point regulator" that allows the stimulation of mitochondrial electron transport without causing self-inflicted oxidative damage. In other words, PGC1 $\alpha$ can turn up the heat safely.

In a second study, the Spiegelman group purified the PGC1 $\alpha$ holoenzyme complex and identified the LRP130 protein, which is mutated in patients with LSFC, as one its components. The authors showed that LRP130 regulates the

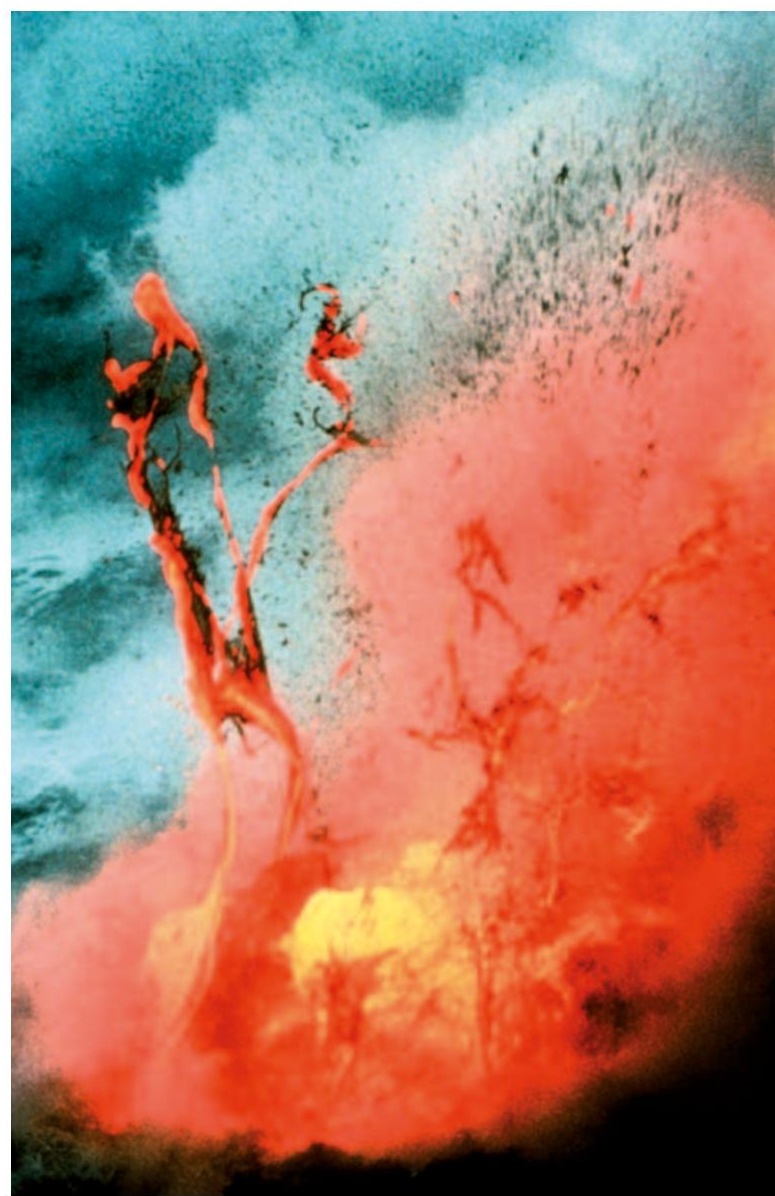

transcription of the Pgc1 $\alpha$ gene, which, in turn, regulates many genes. They found that LRP130 is required for the regulation of certain PGC $1 \alpha$ target genes that are required for gluconeogenesis and mitochondrial electron transport. The significance of these findings was tested in vivo by treating mice with Lrp130 small interfering RNA, and the mice were subsequently either fed or starved. The expression of several gluconeogenesis genes was strongly induced in control mice that had been starved, but this induction was blocked in Lrp130-knockdown mice. Glucose and ATP production were also significantly reduced in these mice compared with control mice. Together, these data link LRP130 and PGC1 $\alpha$ to defective hepatic glucose and energy homeostasis in patients with LSFC. Whether the neurodegeneration phenotype of LSFC is linked to a sensitivity to oxidative stress remains to be determined.

Arianne Heinrichs

ORIGINAL RESEARCH PAPERS St-Pierre, J. et al. Suppression of reactive oxygen species and neurodegeneration by the PGC-1 transcriptional coactivators. Cell 127, 397-408 (2006) | Cooper, M. P. et al. Defects in energy homeostasis in Leigh syndrome French Canadian variant through PGC- $1 \alpha /$ LRP130 complex. Genes Dev. 20, 2996-3009 (2006)

WEB SITE

Bruce M. Spiegelman's laboratory: http://cellbio.med.harvard.edu/ faculty/spiegelman 